\title{
REMARKS ON MOTIVIC HOMOTOPY THEORY OVER ALGEBRAICALLY CLOSED FIELDS
}

\author{
PO HU, IGOR KRIZ AND KYLE ORMSBY
}

\begin{abstract}
We discuss certain calculations in the 2-complete motivic stable homotopy category over an algebraically closed field of characteristic 0 . Specifically, we prove the convergence of motivic analogues of the Adams and AdamsNovikov spectral sequences, and as one application, discuss the 2-complete version of the complex motivic $J$-homomorphism.

MSC: 14F42, 55T15, 19L20 Keywords: Motivic homotopy theory, Adams spectral sequences, $J$-homomorphism
\end{abstract}

\section{InTRODUCTION}

The purpose of this paper is to point out some aspects of the 2-complete stable motivic homotopy category over algebraically closed fields $K$ of characteristic 0 . By this we mean the category of $\mathbb{P}^{1}$-motivic spectra, Bousfield localized at the pushforward of the Moore spectrum $M \mathbb{Z} / 2$. This is, in some sense, the part of motivic stable homotopy theory which is the closest to ordinary topology, although even here, the theory one obtains, as we shall note, contains interesting new phenomena. We shall work in this category throughout this paper, unless specified otherwise. We shall follow the notational convention from [10] (analogous to Real-oriented homotopy theory), which means that for a motivic (generalized) cohomology theory, we write

$$
E^{k+\ell \alpha}(X)=E^{k+\ell, \ell} X
$$

and similarly for homology.

One can then define $B P G L$ and a motivic analog of the Adams-Novikov spectral sequence converging to the 2-completed motivic stable homotopy theory $\pi_{*}^{M o t}$. The $E_{2}$-term is equal to the topological $E_{2}$-term, but with dimensions shifted by twists, and tensored with $\mathbb{Z}[\theta]$ where $\theta$ is the "Tate twist" (see below). The differentials mimic the topological differentials, but there is a difference in twist.

To give an example of peculiarities this can cause, recall [22] that in topology, we have generators

$$
\alpha_{i} \in \operatorname{Ext}_{B P_{*} B P}^{1,2 i}\left(B P_{*}, B P_{*}\right)
$$

(in the notation of [22], our $\alpha_{4 k}$ 's are $\bar{\alpha}_{4 k}$ 's, and our $\alpha_{4 k+2}$ 's are $\alpha_{4 k+2 / 3}$ 's). The elements $\alpha_{4 k+2}, \alpha_{4 k+3}$ support a $d_{3}$ differential, and those are the only differentials the $\alpha$-elements are involved in. The element $\alpha_{1}$ represents the non-zero element in the first stable 2 -stem (=2-completed stable homotopy group), called $\eta$. The $\mathbb{Z}[\eta]$ submodule of stable 2-stems generated by the $\alpha$-elements consists of the elements

The first author was partially supported by NSF grant DMS-0503814. The second author was partially supported by NSA grant 08-1477. The third author was partially supported by NSF grant DMS-0602191. 
"related to the $J$-homomorphism" in the sense of Adams [4] (most are in the image of $J$ ).

In the Motivic Adams-Novikov spectral sequence, we have similar elements

$$
\alpha_{i} \in \operatorname{Ext}_{B P_{*} B P}^{1, i(1+\alpha)}\left(B P_{*}, B P_{*}\right) \Rightarrow \pi_{*}\left(\left(S_{M o t}^{0}\right)_{2}^{\wedge}\right)
$$

Theorem 1. Over an algebraically closed field $K$ of characteristic 0 , the element $\theta$ is a permanent cycle in the $p=2$-motivic Adams spectral sequence. Further, inside the $\alpha$-family, the elements (1) for $i \equiv 0,1 \bmod 4$ are permanent cycles in the motivic Adams-Novikov spectral sequence at $p=2$, representing elements $a_{4 k} \in \pi_{4 k-1+4 k \alpha}^{M o t}\left(\left(S_{M o t}^{0}\right) \hat{2}\right)$, resp. $a_{4 k+1} \in \pi_{4 k+(4 k+1) \alpha}^{M o t}\left(\left(S_{M o t}^{0}\right) \hat{2}\right)$. Write $\eta=\alpha_{1} \in$ $\pi_{\alpha}\left(\left(S^{0}\right)_{2}^{\wedge}\right)$.

Then

$$
a_{4 k} \eta^{m} \neq 0, a_{4 k+1} \eta^{m} \neq 0
$$

for any positive integers $k, m$, while

$$
a_{4 k} \eta^{3} \theta=a_{4 k+1} \eta^{3} \theta=\eta^{4} \theta=0 .
$$

Outside the $\alpha$-family, the element $\alpha_{1}^{2} \eta_{3 / 2} \in \operatorname{Ext}_{B P G L_{*}}^{5,15(1+\alpha)}$ represents a non-zero element

$$
x \in \pi_{10+15 \alpha}^{M o t}\left(\left(S_{M o t}^{0}\right)_{2}^{\wedge}\right)
$$

which maps to 0 in etale homotopy theory, and satisfies

$$
\theta x=0 .
$$

Remark: Note that computing Morel's Milnor-Witt ring [18], one easily sees that $\eta^{m} \neq 0$.

Comment: We will see (Lemma 2) that in each twist, the homotopy groups of the 2-completion of the motivic sphere spectrum are in fact the homotopy groups of the 2-completion of an (ordinary topological) bounded below spectrum, whose homotopy groups are the homotopy groups of the motivic sphere spectrum. Therefore, by Bousfield [5], we indeed have a short exact sequence

$$
\begin{aligned}
& 0 \rightarrow \operatorname{Ext}^{1}\left(\mathbb{Z} / 2^{\infty}, \pi_{m+n \alpha}\left(S_{M o t}^{0}\right)\right) \rightarrow \pi_{m+n \alpha}\left(\left(S_{M o t}^{0}\right)_{2}^{\wedge}\right) \\
& \rightarrow \operatorname{Hom}\left(\mathbb{Z} / 2^{\infty}, \pi_{m-1+n \alpha}\left(S_{\text {Mot }}^{0}\right)\right) \rightarrow 0 .
\end{aligned}
$$

2-adic etale stable homotopy theory over an algebraically closed field turns out to be just topological 2-adic stable homotopy theory $\otimes \mathbb{Z}\left[\theta, \theta^{-1}\right]$. Because of this, Theorem 1 gives an example where motivic stable homotopy groups of spheres substantially deviate from the topological case, by producing an element which "forgets" to 0 .

Prompted by a suggestion of Dan Isaksen [12], we also investigate the "complex" motivic $J$-homomorphism in the present context. We may consider the $\mathbb{A}^{1}$-space of stable self-equivalences of $S_{M o t}^{0}$. This space can be constructed as follows: ?^ $S^{n(1+\alpha)}$ on the category of based $\mathbb{A}^{1}$-spaces has a right Quillen adjoint, which we denote by $\Omega^{n(1+\alpha)}$. Then we have

$$
Q_{M o t} S^{0}=\text { hocolim } \Omega^{n(1+\alpha)} S^{n(1+\alpha)} .
$$

Note by Morel [18] that

$$
\pi_{0}\left(Q_{M o t} S^{0}\right)=G W(K) .
$$


The right hand side, which is called the Grothendieck-Witt ring, is $\mathbb{Z}$ when $K$ is algebraically closed. Now while in general there is no map from an $\mathbb{A}^{1}$-space $X$ to $\pi_{0}(X)$ (think about $X=\mathbb{G}_{m}$ ), there is a degree map from $Q_{M o t} S^{0}$ to the motivic Eilenberg-MacLane space representing 0 'th integral cohomology, which is equivalent to $\mathbb{Z}$. Therefore, for our purposes we may define $F_{M o t}$ simply as the fiber of the map deg: $Q_{M o t} S^{0} \rightarrow \mathbb{Z}$ over the subset $\{+1,-1\}$. Let $G L_{M o t}$ be the direct limit of the group schemes $G L_{n}$. Then, as in topology, we have a map

$$
\mathcal{J}: G L_{M o t} \rightarrow F_{M o t}
$$

Now let us pass to homotopy groups. The $(m+n \alpha)$ 'th homotopy group of the right hand side of (3) is the stable homotopy group $\pi_{m+n \alpha} S_{M o t}^{0}$ for $m>0$. On the other hand, the $(m+n \alpha)^{\prime}$ th homotopy group of the left hand side is the algebraic $K_{m-n+1}$-group of the ground field $K$. Obviously, this can be non-zero only when $m-n>0$, and additionally we must have $m, n \geq 0$. After reindexing, we get a map for all $k, \ell \geq 0$,

$$
J: K_{k}(K) \rightarrow \pi_{(k-1)+\ell(1+\alpha)} S^{0} .
$$

This is (the "complex version") of the motivic $J$-homomorphism. To put this in our context, we would like a "2-complete version" of the map (4). One complication is that it is at present not known how to realize (3) as a map of ( $\mathbb{P}^{1}$-stable) spectra, since $F_{M o t}$ should be the infinite loop space associated with the multiplicative spectrum of the sphere spectrum, but no infinite loop space machine is known for $\mathbb{P}^{1}$-stable spectra, so this is not known. For the moment, we recall [18] that there is a canonical pushforward functor $P F$ from the category simplicial sets to the category of Morel-Voevodsky $\mathbb{A}^{1}$-spaces, which possesses a right adjoint, which we shall denote by $F P$. Further, $P F$ obviously preserves cofibrations and acyclic cofibrations, so this is a Quillen adjunction. There is a similar Quillen adjoint pair $P F_{s}, F P_{s}$ between the category of spectra and $\mathbb{P}^{1}$-stable motivic spectra. Since $P F_{s}$ turns shift desuspensions of suspension spectra to shift desuspension of suspension spectra on $P F$, by commutation of adjoints, we have, for a $\mathbb{P}^{1}$-stable motivic spectrum $E$, and an integer $n$,

$$
F P_{s}(E)_{n} \simeq F P\left(E_{n}\right) .
$$

Now we can "2-complete" (3) by applying $F P \Omega^{\ell \alpha}$ to both sides and then 2-complete in the category of spaces (=simplicial sets). We claim that for $k+\ell>1$, this gives a map

$$
\pi_{k}\left(K^{a l g}(K)_{2}^{\wedge}\right) \rightarrow \pi_{(k-1)+\ell(1+\alpha)}\left(\left(S_{M o t}^{0}\right)_{2}^{\wedge}\right) .
$$

Indeed, since the 2-completion of the 0-connected component of the infinite loop space of a spectrum (in the category spaces) is the 0 -component of the infinite loop space of the 2-completion of the spectrum, it suffices to prove the following

Lemma 2. The functor FPs preserves 2-completion on the level of homotopy categories.

We will prove this in section 5 below. Now it is also known by a calculation of Suslin [23] that the left hand side of $(6)$ is $\mathbb{Z}_{2}$ when $k$ is even and 0 otherwise. On the other hand, as we shall see below, the image of (6) only depends on $k+2 \ell-1$. In Section 5, we shall prove the following result 
Theorem 3. The image of (6) is isomorphic to the 2-primary component of the image of the (ordinary complex) $J$-homomorphism in dimension $k+2 \ell-1$.

In order to prove Theorem 1, we need to know the structure of algebraic cobordism over an algebraically closed field. This in turn needs the Adams spectral sequence, which needs the algebra of bistable operations in motivic cohomology (the motivic Steenrod algebra). This calculation was done by Voevodsky in the late 90 's, but not published at the time of the writing of this note. ${ }^{1}$ To make the present note self-contained, we give the computation here in the case of an algebraically closed field (although the method works in greater generality). Namely, by investigating the structure of symmetric products, we prove

Theorem 4. (Voevodsky) Let $K$ be an algebraically closed field of characteristic 0 . Denoting by $H^{M o t}$ the $H \mathbb{Z} / 2$-motivic (co)homology spectrum over $K$, and by $\theta$ Tate twist (of cohomological dimension $\alpha-1$ ), the algebra of bistable operations $H^{\text {Mot* }} H^{\text {Mot }}$ is generated as a $\mathbb{Z} / 2[\theta]$-module by reduced power operations $P^{s}$ (of dimension $s(1+\alpha)$ ) and the Bockstein (of dimension 1).

By [25], we therefore have

Corollary 5. The dual motivic Steenrod algebra over an algebraically closed field $K$ is given by

$$
H_{*}^{M o t} H^{M o t}=\mathbb{Z} / 2\left[\theta, \tau_{0}, \tau_{1}, \tau_{2}, \ldots, \xi_{1}, \xi_{2}, \ldots\right] /\left(\tau_{i}^{2}=\xi_{i+1} \theta\right)
$$

where the dimensions of $\xi_{i}, \tau_{i}, \theta$ are $\left(2^{i}-1\right)(1+\alpha),\left(2^{i}-1\right)(1+\alpha)+1,1-\alpha$, respectively.

Let a cell spectrum of finite type in the $\mathbb{P}^{1}$-stable motivic category be a spectrum which can be obtained by successively attaching cells (=cones on spheres) in dimensions $m+n \alpha, m, n \geq 0$, with only finitely many cells in dimension $m+n \alpha$ for each given $m$.

Theorem 6. Over an algebraically closed field of characteristic 0 , the Adams spectral sequence

$$
\operatorname{Ext}_{H_{*}^{M o t} H^{M o t}}\left(H_{*}^{M o t}(X), H_{*}^{M o t}\right) \Rightarrow \pi_{*}\left(X_{2}^{\wedge}\right)
$$

converges for any cell spectrum $X$ of finite type.

The proof will be given in the Appendix (Section 6).

The algebraic cobordism spectrum is a positive cell spectrum of finite type (using Schubert cells). Using this, we can calculate the 2-completed algebraic cobordism groups:

Theorem 7. Over an algebraically closed field $K$, the 2-completed algebraic cobordism groups are given by

$$
\left(M G L_{2}^{\wedge}\right)_{*}=\mathbb{Z}_{2}\left[\theta, v_{1}, v_{2}, \ldots, u_{i} \mid i \neq 2^{n}-1\right]
$$

where $v_{n}$ has dimension $\left(2^{n}-1\right)(1+\alpha)$ and the $u_{i}$ have dimension $i(1+\alpha)$.

Completed at 2 , one can next construct $B P G L$ by mimicking, again, the construction from topology. Using this, we can then conclude

\footnotetext{
${ }^{1}$ After this note was written, [26] appeared on the $K$-theory archive. That manuscript was not available when the first version of the present note was written, which is why we were compelled to obtain an independent proof.
} 
Theorem 8. There exists a convergent Adams-Novikov spectral sequence

$$
\operatorname{Ext}_{B P G L_{*} B P G L}\left(B P G L_{*}, B P G L_{*}\right) \Rightarrow \pi_{*}\left(\left(S_{M o t}^{0}\right)_{2}^{\wedge}\right) \text {. }
$$

The present note is organized as follows: In Section 2, we discuss the main technical tool of our approach to Voevodsky's result, the motivic transfer. In Section 3 , we apply this technique to analyzing the motivic cohomology of the symmetric smash-powers of spheres. In Section 4, we prove our main theorems and discuss the motivic Adams-Novikov spectral sequence. The Proof of Theorem 3 will be done in Section 5 .

Acknowledgements: Since the first version of this paper was posted on the $K$ theory archive, the paper [8], the results of which partially (but not fully) overlap with ours, appeared on arXiv. We thank Dan Dugger for a subsequent discussion, which prompted us to make some clarifications in the present version. We also wish to thank Fabien Morel and Mike Hopkins for valuable discussions.

\section{The motivic Elmendorf construction}

In this paper, we discuss symmetric products of varieties, which are generally not smooth, so we work with the cd-h topology in finite schemes over a field $k$.

Definition: A 1-point compactification $X^{*}$ of a quasiprojective variety $X$ embedded into a projective variety $\bar{X}$ is $\bar{X} /(\bar{X}-X)$.

Example: $S^{n(1+\alpha)}$ is a 1-point compactification of $\mathbb{A}^{n}$. $\operatorname{Quot}_{\Sigma_{d}} \bigwedge_{d} S^{n(1+\alpha)}$ is a 1-point compactification of Quot $_{\Sigma_{d}} \mathbb{A}^{\text {nd }}$. Here on the right hand side, Quot $_{G}$ is the functor which on a $G$-equivariant scheme $X$ takes the value $X / G$ in the category of schemes, and commutes with direct limits.

We should note that if $X$ is smooth and $\bar{X}-X=D_{1} \cup \ldots \cup D_{m}$ where $D_{i}$ are divisors with normal crossings, then denoting by $Q$ the poset of non-empty subsets of $\{1, \ldots, m\}$ with respect to inclusion, the natural map

$$
\underset{S \in Q}{\operatorname{hocolim}} \bigcap_{i \in S} D_{i} \rightarrow \bar{X}-X
$$

is an equivalence (by iterated use of the cdh-diagram), which gives a model of the 1-point compactification in the smooth category with Nisnievich topology.

Lemma 9. Let $X^{*}$ be a 1-point compactification of a smooth quasiprojective variety $X$ of dimension $n$. Then, in the $\mathbb{A}^{1}$-stable category,

$$
X^{*} \simeq \Sigma^{n(1+\alpha)} D\left(X^{\xi}\right)
$$

where $D$ denotes Spanier-Whitehead dual and $X^{\xi}$ denotes the Thom spectrum of $X$ with respect to its virtual normal bundle of dimension 0. 
Proof: This is an example of a purity result. It can be proved, for example, by the methods of [11].

Now the main point of this section is to consider "smooth models" of quotients of smooth varieties by finite groups $G$. In topology, there is the following method, known as the Elmendorf construction: The orbit category $\mathfrak{O}$ is the category of $G$ orbits and $G$-equivariant maps. Equivalently, it is the category of subgroups and outer subconjugacies (i.e. subconjugacies modulo inner conjugacies in the target group). Then the fixed points $X^{\text {? }}$ of a space $X$ under subgroups of $G$ form a contravariant functor

$$
\mathfrak{O} \rightarrow \text { Spaces } .
$$

An example of a covariant functor on the orbit category is $G /$ ?. Another example of a covariant functor is $E \mathcal{F}_{\text {? }}$ where $E \mathcal{F}_{H}$ is a $G$-CW complex characterized up to $G$-homotopy equivalence by the property that

$$
\left(E \mathcal{F}_{H}\right)^{K} \simeq \begin{cases}* & \text { for } K \text { subconjugate to } H \\ \emptyset & \text { else. }\end{cases}
$$

One can make $E \mathcal{F}_{\text {? }}$ into a covariant functor on the orbit category, for example by taking the 2 -sided bar construction

$$
E \mathcal{F}_{?}=B(*, \mathfrak{O}, \mathfrak{O} / ?)
$$

(Here $\mathfrak{O} / H$ denotes the contravariant functor which assigns to $K$ the set of morphisms $K \rightarrow H$ in $\mathfrak{O}$.) The notation $E \mathcal{F}_{H}$ is explained by noting that the set $\mathcal{F}_{H}$ of subgroups subconjugate to $H$ is an example of a family, i.e. a set of subgroups of $G$ closed under subconjugacy. Analogously to (7), one can define the classifying space of any family. Then, in topology, the natural map

$$
E \mathcal{F}_{?} \times_{\mathfrak{O}} X^{?} \rightarrow X / G
$$

is an equivalence for any $G$-CW complex $X$.

In $\mathbb{A}^{1}$-homotopy, $(7)$ does not characterize $E \mathcal{F}_{H}$ up to $G$ - $\mathbb{A}^{1}$-equivalence, and the construction (8) is usually wrong for the purposes of (9). An alternative construction which will work in the case we are interested in (the symmetric products) is obtained as follows: Let $G$ act effectively on $d=\{1, \ldots, d\}$. Then set

$$
E \mathcal{F}_{H}=\mathbb{A}^{d \infty}-\bigcup_{K \notin \mathcal{F}_{H}}\left(\mathbb{A}^{d \infty}\right)^{K} .
$$

In fact, we also want to adapt (9) to take the form

$$
E \mathcal{F}_{?} \times_{(\mathfrak{O})} X^{?} \rightarrow \operatorname{Quot}_{G}(X) .
$$

Denote

$$
N(H, K)=\left\{g \in G \mid g^{-1} H g \subseteq K\right\} .
$$

Then on the left hand side of (11), we mean the obvious coequalizer

$$
\coprod_{(H),(K)} \operatorname{Quot}_{W_{G}(H)} \underset{N(H, K) / H}{\amalg}\left(E \mathcal{F}_{H} \times X^{K}\right) \underset{(H)}{\longrightarrow} \operatorname{Quot}_{W_{G}(H)}\left(E \mathcal{F}_{H} \times X^{H}\right)
$$


where $W_{G}(H)$ denotes the Weyl group of $H$ in $G$, and the coproducts on the right (resp. left) side of (12) are over conjugacy classes of subgroups (resp. subconjugate pairs of conjugacy classes of subgroups of $G$ ). The key point is that one can construct inductively the subspace $Y_{\mathcal{F}}$ of the construction (12) on a family $\mathcal{F}$ of subgroups of $G$ : for $H \notin \mathcal{F}$, such that every proper subgroup of $H$ is in $\mathcal{F}$, to construct $Y_{\mathcal{F} \cup(H)}$, one attaches the subspace

$$
E \mathcal{F}_{H} \times\left(\bigcup X^{K}\right)
$$

to $Y_{\mathcal{F}}$ according to the prescribed identification given by (12). When $\mathcal{F}$ is the family of all subgroups of $G, Y_{\mathcal{F}}$ is the left hand side of (12).

For any construction of $E \mathcal{F}_{H}$, one then has (by induction on the strata)

Lemma 10. Let $X^{>H}=\bigcup_{K \notin \mathcal{F}_{H}} X^{K}$. If the collapse map

$$
E \mathcal{F}_{H+} \wedge\left(X^{H} / X^{>H}\right) \rightarrow X^{H} / X^{>H}
$$

is an $H$-A $\mathbb{A}^{1}$-equivalence, then (11) is an $\mathbb{A}^{1}$-equivalence. In fact, more generally, for any subgroup $\Gamma \subseteq G$, the natural map

$$
\left(\Gamma \backslash G / ? \times E \mathcal{F}_{?}\right) \times_{(\mathfrak{O})} X^{?} \rightarrow \operatorname{Quot}_{\Gamma}(X)
$$

is a $\Gamma$ - $\mathbb{A}^{1}$-equivalence.

Corollary 11. Under the assumptions of Lemma 10, let $\mathfrak{H}(X)$ denote Bloch's Chow chain complex on $X$ (resp. its natural extension to $G-\mathbb{A}^{1}$-spaces). Then the transfer maps associated with free group action for each subgroup $H$

$$
t: \mathfrak{H}\left(\operatorname{Quot}_{W(H)} E \mathcal{F}_{?} \times X^{?}\right) \rightarrow \mathfrak{H}\left(\operatorname{Quot}_{W(H)}\left(\Gamma \backslash G / ? \times E \mathcal{F}_{?}\right) \times X^{?}\right)
$$

multiplied by the multiplicity factors

$$
\left|\pi_{H}(\Gamma \cap N(H))\right|
$$

where $\pi_{H}: N(H) \rightarrow W(H)=N(H) / H$ is the projection fit together via (14) to define a transfer map in the stable category

$$
t: \mathfrak{H}\left(\operatorname{Quot}_{G}(X)\right) \rightarrow \mathfrak{H}\left(\operatorname{Quot}_{\Gamma}(X)\right) .
$$

Further, there exists a finite filtration on $\mathfrak{H}\left(Q u o t_{\Gamma}(X)\right)$ such that the associated graded map of the composition

$$
\mathfrak{H}\left(\operatorname{Quot}_{\Gamma} X\right) \rightarrow \mathfrak{H}\left(\operatorname{Quot}_{G} X\right) \rightarrow \mathfrak{H}\left(\operatorname{Quot}_{\Gamma} X\right)
$$

is multiplication by $|G / H|$.

In the last sentence of the Corollary, the filtration is supplied by totally ordering the poset of subconjugacy classes of $\mathrm{G}$, and could probably be eliminated by more careful consideration, but it does not matter for our purposes. 


\section{Symmetric PRODUCTS}

In this section, we will investigate the following example:

$$
X=\bigwedge_{d} S^{n(1+\alpha)}
$$

where $\Sigma_{d}$ acts by permutation of coordinates. We are essentially interested in mimicking the computation of the cohomology of symmetric products of spheres by Nakaoka [20] in the motivic situation. We claim that condition (13) is satisfied when we set (10) with $\Sigma_{d}$ acting on $d$ by the standard permutation representation.

First of all, we note that the strata $X^{H} / X^{>H}$ are only non-trivial when

$$
H=\Sigma_{d_{1}} \times \ldots \times \Sigma_{d_{k}},
$$

$d_{1}+\ldots+d_{k}=d$ where $\Sigma_{d_{i}}$ acts on $d_{i}$ by the standard permutation representation. Then $X^{H} / X^{>H}$ is the 1-point compactification of the pure stratum

$$
X^{H}-X^{>H}=\mathbb{A}^{n k}-\Delta
$$

where $\Delta$ is the big diagonal (the union of elements with 2 or more coordinates coinciding). Using (10) and (17), we can then define an $\mathbb{A}^{1}$-homotopy inverse

$$
\iota: \mathbb{A}^{n k}-\Delta \rightarrow E \mathcal{F}_{H} \times\left(\mathbb{A}^{n k}-\Delta\right)
$$

of the natural projection

$$
p: E \mathcal{F}_{H} \times\left(\mathbb{A}^{n k}-\Delta\right) \rightarrow \mathbb{A}^{n k}-\Delta
$$

( $p$ collapses the first coordinate to a point). To define $\iota$, use $I d$ for the second coordinate in the target of (18). For the first coordinate, we need a map

$$
\mathbb{A}^{n k}-\Delta \rightarrow \mathbb{A}^{d \infty}-\bigcup_{K \notin \mathcal{F}_{H}}\left(\mathbb{A}^{d \infty}\right)^{K}
$$

But this is obvious: simply send

$$
\begin{aligned}
& \left(\left(x_{11}, \ldots, x_{n 1}\right), \ldots,\left(x_{1 k}, \ldots, x_{n k}\right)\right) \\
& \mapsto\left(\left(x_{11}, \ldots x_{n 1}, 0,0, \ldots\right)^{d_{1}}, \ldots,\left(x_{1 k}, \ldots, x_{n k}, 0,0, \ldots\right)^{d_{k}}\right) \in\left(\mathbb{A}^{\infty}\right)^{d} .
\end{aligned}
$$

By definition, (19) is strictly left inverse to (18). To construct an $\mathbb{A}^{1}$-homotopy

$$
i p \simeq I d,
$$

first recall that we have the "Milnor trick" homotopy

$$
\begin{gathered}
k_{t}: \mathbb{A}^{\infty} \rightarrow \mathbb{A}^{\infty}, \\
k_{t}\left(x_{1}, x_{2}, \ldots\right)=(1-t)\left(x_{1}, x_{2}, \ldots\right)+t\left(0, \ldots, 0, x_{1}, 0, \ldots, 0, x_{2}, \ldots\right)
\end{gathered}
$$

( $n$ 0's inserted before each coordinate). Clearly, $\left(k_{t}\right)^{d}$ restricts to an $H$-homotopy

$$
\ell_{t}: E \mathcal{F}_{H} \rightarrow E \mathcal{F}_{H}
$$

where $\ell_{0}=I d, \ell_{1} \subseteq(0 \times 0 \times \ldots 0 \times \mathbb{A} \times 0 \times \ldots \times 0 \times \mathbb{A} \times \ldots)^{d}$. (20) then follows if we can construct a homotopy

$$
i p \simeq \ell_{1} \text {. }
$$

But for this purpose, we may now simply use

$$
t(i p)+(1-t) \ell_{1},
$$

which completes the proof of our claim. 
Now we see that an $\mathbb{A}^{1}$-homotopy inverse for the map in the assumption of Lemma 10 is in our case obtained simply by 1-point compactifying (18), (20) in the $\mathbb{A}^{n k}-\Delta$ coordinate. We obtain

Proposition 12. Let $S \subset \Sigma_{d}$ be the 2-Sylow subgroup obtained by the standard permutation representation of a product of wreath products of copies of $\mathbb{Z} / 2$. Then there exists for $X$ in (15) a transfer map

$$
\tau: \mathfrak{H}\left(\operatorname{Quot}_{\Sigma_{d}}(X)\right) \rightarrow \mathfrak{H}\left(X^{\prime}=\operatorname{Quot}_{S}\left(\bigwedge_{d} S^{n(1+\alpha)}\right)\right)
$$

such that if we denote by $p: \mathfrak{H}\left(X^{\prime}\right) \rightarrow \mathfrak{H}\left(\right.$ Quot $\left._{\Sigma_{d}}(X)\right)$ the natural projection, then $\tau p$ is a 2-complete equivalence.

Proof: By Corollary 11, there is a filtration such that on the associated graded pieces, $\tau p$ is the multiplication by the odd number $\left|\Sigma_{d} / S\right|$.

Because of Proposition 12, it now makes sense to examine the motivic (co)homology of $X^{\prime}$ in what we call the stable range. This means that for any given constant $k$, we may choose $n$ sufficiently large, and consider dimensions

$$
i+j \alpha
$$

where $(2 n \leq) i+j \leq 2 n+k$. Let us denote $X^{\prime}$ by $X^{\prime}(n)$ when we wish to express dependence on $n$.

Definition: By a stratification of an $\mathbb{A}^{1}$-space we mean an increasing filtration $F_{i} X$ such that $F_{0} X=\emptyset, F_{N} X=X$, such that for each $i$ there exists a smooth quasiprojective variety $V_{i}$ such that $F_{i} X / F_{i-1} X$ is a one point compactification of $V_{i}$. We refer to the $V_{i}$ 's as the pure strata.

Proposition 13. There exists a stratification of $X^{\prime}(n)$ where there are canonical injections from the set of pure strata of $X^{\prime}(n)$ to the set of pure strata of $X^{\prime}(n+1)$, and all sequences of pure strata related by these injections are of one of the following forms:

$$
\left(\mathbb{G}_{m}\right)^{\times \ell} \times \mathbb{A}^{n+k}, \quad k \text { independent of } n,
$$

or

$$
\text { An } \mathbb{A}^{m} \text {-bundle } V \text { on a smooth variety } Y \text { where } m
$$
tends to $\infty$ as $n$ does.

Furthermore, strata of the form (21) do not occur unless $d=2^{k}$ for some integer $k$.

Proof: Induction. It suffices to consider $d=2^{k}$, since otherwise $X^{\prime}$ is the smashproduct of the cases for $2^{k_{i}}$ where $k_{i}$ are places of the 1's in the binary expansion of $d$.

Now the case $k=1$ is obvious, so consider

$$
X^{\prime}=\operatorname{Quot}_{\mathbb{Z} / 2}\left(X^{\prime \prime} \wedge X^{\prime \prime}\right)
$$

where $X^{\prime \prime}$ is stratified as in the statement of the Proposition. In the rest of the proof, all quotients are taken in the category of schemes, so we dispense with the Quot 
notation accordingly. So first, we take the stratification of $X^{\prime \prime} \wedge X^{\prime \prime}$ by the smashproduct of two copies of the stratification of $X^{\prime \prime}$, and then take a $\mathbb{Z} / 2$-quotient. If the pure strata of $X^{\prime \prime}$ are $A_{1}, \ldots, A_{N}$, then this gives pure strata of (23) of the form

$$
\begin{aligned}
& A_{i} \times A_{j}, i<j, \\
& \left(A_{i} \times A_{i}\right) /(\mathbb{Z} / 2)
\end{aligned}
$$

$\left((25)\right.$ is taken in the scheme theoretical sense, so it is really $\left.\operatorname{Quot}_{\mathbb{Z} / 2}\left(A_{i} \times A_{i}\right)\right)$. The strata (24) are clearly of the form (22), the strata (25) may need to be further stratified.

More concretely, if $A_{i}$ is of the form (22), we may stratify (25) by taking the bundle $\operatorname{Sym}^{2}(V)$ on $Y$, and then the $\mathbb{Z} / 2$-quotient of the bundle induced from $V \times V$ on $(Y \times Y)-Y$. Both are clearly of the form (22).

Suppose, then, that $A_{i}$ is of the form (21). Once again, then, we have the pure stratum

$$
\left(\left(\mathbb{G}_{m}\right)^{\times \ell} \times\left(\mathbb{G}_{m}\right)^{\times \ell}\right)-\left(\left(\mathbb{G}_{m}\right)^{\times \ell}\right) \times_{\mathbb{Z} / 2}\left(\mathbb{A}^{n+k} \times \mathbb{A}^{n+k}\right),
$$

which is of the form (22). What we are left with is

$$
\left(\mathbb{G}_{m}\right)^{\times \ell} \times \operatorname{Sym}^{2}\left(\mathbb{A}^{n+k}\right) .
$$

We stratify the second coordinate of (27) by taking as the $j$ 'th stratum the $\mathbb{Z} / 2$ quotient of the subspace of

$$
\mathbb{A}^{n+k} \times \mathbb{A}^{n+k}
$$

consisting of pairs of points whose respective coordinates coincide except the first $j$. Assuming the characteristic of the ground field is not 2, the bottom pure stratum is

$$
\mathbb{A}^{n+k},
$$

the higher strata are

$$
\mathbb{G}_{m} \times \mathbb{A}^{n+k+i},
$$

leading to strata of (27) of type (21), as claimed.

The relationship between symmetric products and the motivic cohomology spectrum is proved in [24]. Essentially, we have a filtration on the motivic Eilenberg-Mac Lane spaces in dimension $n(1+\alpha)$ such that the associated graded pieces are (15). We derive here one more consequence of the above methods which will be useful later.

Proposition 14. The motivic Eilenberg-Mac Lane spectrum $H \mathbb{Z} / 2^{M o t}$ is cell (i.e. can be obtained by successivelly attaching cones to $k+\ell \alpha$-dimensional homotopy classes, see the Appendix).

Proof: Since the motivic Eilenberg-Mac Lane spectrum is equivalent to the homotopy direct limit of the motivic (bistable) suspension spectra of $n(1+\alpha)$-dimensional Eilenberg-MacLane spaces, which in turn can be constructed by successively attaching symmetric products of the form (15), it suffices to prove the statement for the direct limit under $n$ of the suspension spectra of the symmetric quotients of the spaces (15). By further filtering with respect to fixed point, we can next pass to 1-point compactifications of the unordered configuration spaces of $d$ points in $\mathbb{A}^{n}$. Since $H \mathbb{Z} / 2^{M o t}=H \mathbb{Z} \wedge M \mathbb{Z} / 2$ is 2 -complete (note that $[Z, M \mathbb{Z} / 2 \wedge X]$ coincides, 
up to suspension, with $[Z \wedge M \mathbb{Z} / 2, X])$, we can additionally work in the 2-complete motivic category, so we can take advantage of transfer in (motivic) stable homotopy and work with the suspension spectra of the 1-point compactification $\Phi$ of the quotient of the ordered configuration space of $d$ points in $\mathbb{A}^{n}$ by a product $P$ of iterated wreath products of copies of $\mathbb{Z} / 2$, which is a 2-Sylow subgroup of $\Sigma_{d}$. (Recall that, up to equivalence, a wedge summand of a cell spectrum is cell by the "Eilenberg swindle", although such a claim is false for finite cell objects.)

To describe the motivic transfer in more detail, there are many versions, but for the present purpose, the following will do. The ordered configuration space $X$ of $d$ points in $\mathbb{A}^{n}$ is an affine variety (otherwise, one would use the 'affinization'). We are trying to construct a transfer map

$$
\Sigma^{N(1+\alpha)}\left(\left(X / \Sigma_{d}\right)^{*}\right) \rightarrow \Sigma^{N(1+\alpha)}\left((X / P)^{*}\right)
$$

for $N>>0$. To this end, choose a closed embedding

$$
j: X / P \rightarrow \mathbb{A}^{N} \text {. }
$$

Consider then the closed embedding

$$
j \times p: X / P \rightarrow \mathbb{A}^{N} \times X / \Sigma_{d} .
$$

Then consider the induced map

$$
\left(\mathbb{A}^{N} \times X / \Sigma_{d}\right)^{*} \rightarrow\left(\mathbb{A}^{N} \times X / \Sigma_{d}\right)^{*} /\left(\mathbb{A}^{N} \times X / \Sigma_{d}-\operatorname{Im}(j \times p)\right)^{*} .
$$

In the general case, we would invoke purity here to identify the right hand side of (31) with the based Thom space on $(X / P)^{*}$, but in the present case, it is actually easy to see that the canonical map

$$
\left(\mathbb{A}^{N} \times X / P\right) \rightarrow \mathbb{A}^{N} \times X / \Sigma_{d}
$$

given by $(a, x) \mapsto(a+j(x), p(x))$ induces an isomorphism from

$$
\left(\mathbb{A}^{n} \times X / P\right)^{*} /\left(\mathbb{A}^{N}-\{0\} \times X / P\right)^{*}
$$

to the right hand side of (31). Using this isomorphism, (31) becomes (30).

But by the general principle that a finite union is a homotopy direct limit of the diagram of intersections, $\Phi$ is further a homotopy direct limit of spaces of the form $X^{\prime}$ in Proposition 12. For such spaces, in turn, the suspension spectrum is cell by the proof of Proposition 13.

Note that strata of the form (22) may be neglected stably - it is easy to see that the stabilization maps of Proposition 13 on these strata are 0 (because $m \rightarrow \infty$ ). Note also that any considerations using the cdh-category here can be modelled in the smooth category using the comment under the Example in the beginning of Section 2.

\section{Proofs of the theorems and the motivic Adams-Novikov spectral SEQUENCE}

Now although the statement of Proposition 13 is precisely as needed for the induction, note that the proof actually precisely accounts for the strata of type 
(21), showing that the motivic $\mathbb{Z} / 2$-cohomology

$$
H_{M o t}^{*}\left(X^{\prime}, \mathbb{Z} / 2\right), X^{\prime}=\operatorname{Quot}_{S}\left(\bigwedge_{d} S^{n(1+\alpha)}\right),
$$

in the stable range is the subspace of $\mathbb{Z} / 2$-valued etale cohomology which is the tensor product over $\mathbb{Z} / 2$ of $\mathbb{Z} / 2[\theta]$ with the $\mathbb{Z} / 2$-module with basis

$$
Q_{k} \ldots Q_{1} \alpha
$$

where $\alpha$ is the characteristic class of dimension $n(1+\alpha)$, and $Q_{i}$ is either of the form $P^{s}$ or $\beta P^{s}$ where $P^{s}$ is of dimension $s(1+\alpha)$, and $\beta$ is of dimension 1 . Now recall Proposition 12. Passing from the free $\mathbb{Z} / 2$-module on (34) to the cohomology of $X$ amounts to taking a certain direct summand. Passing to etale cohomology amounts to inverting $\theta$, but also in the etale cohomology one knows that the cohomology is the same as in the topological situation, tensored with $\mathbb{Z} / 2\left[\theta, \theta^{-1}\right]$. Thus, in etale cohomology, the direct summand is obtained by imposing the Adem relations. But the Adem relations respect twist, and so we see that the summand of (33) corresponding to the cohomology of $X$ (in the stable range) is generated, as a $\mathbb{Z} / 2[\theta]$-module, by admissible words of the form (34). Dimensional accounting ([25]) shows that the elements $P^{s}, \beta P^{s}$ we constructed must, in fact, be the reduced power operations and their Bocksteins. Thus, we have proved Theorem 4, and hence Corollary 5.

Let us now turn to Theorem 7. By Theorem 4 and [17], we know that the motivic Adams spectral sequence in our situation converges to the homotopy of $M G L$ (consider finite Thom spectra and pass to direct limit - convergence follows from the fact that the homotopy will eventually stabilize, i.e. remain constant, in dimensions $k+\ell \alpha$ with $k+\ell<N, N$ increasing).

Now (recall that we are over an algebraically closed field) one has ([6])

$$
H \mathbb{Z} / 2_{*}^{M o t} M G L=\mathbb{Z} / 2\left[\xi_{1}, \xi_{2}, \ldots\right] \otimes \mathbb{Z} / 2\left[m_{i} \mid i \neq 2^{k}-1\right]
$$

as a comodule over $H \mathbb{Z} / 2_{*}^{M o t} H \mathbb{Z} / 2^{M o t}\left(m_{i}\right.$ are primitive, $i \neq 2^{k}-1$ ), by arguments which parallel exactly the topological case. Now using the Adams spectral sequence, Theorem 7 follows.

Therefore, over an algebraically closed field $K$ of characteristic 0 , we have formal group law theory for algebraically oriented spectra, which parallels the topological case (see also $[14,15,16]$ ). In particular, we have the Quillen idempotent, and $M G L_{2}^{\wedge}$ is a wedge of suspensions of copies of a spectrum $B P G L$ where

$$
B P G L_{*}=\mathbb{Z}_{2}\left[\theta, v_{1}, v_{2}, \ldots\right], \quad \operatorname{dim}\left(v_{k}\right)=\left(2^{k}-1\right)(1+\alpha) .
$$

Following arguments of Adams [1], we then see that the motivic analogue of the Adams-Novikov spectral sequence also converges to 2-completed stable homotopy groups. One also has an isomorphism of Hopf algebroids

$$
\left(B P G L_{*}, B P G L_{*} B P G L\right) \cong\left(B P_{*}, B P_{*} B P\right) \otimes_{\mathbb{Z}_{2}} \mathbb{Z}_{2}[\theta]
$$

where the Tate twist is primitive. Therefore, an analogous relation is true for the ANSS $E_{2}$-terms:

$$
\operatorname{Ext}_{B P G L_{*}}^{s, t(1+\alpha)+n(1-\alpha)}= \begin{cases}E x t_{B P_{*}}^{s, 2 t} & \text { for } n \geq 0 \\ 0 & \text { for } n<0\end{cases}
$$


Now to prove Theorem 1, we need a device for comparing differentials to the topological case. In characteristic 0, we know that maps of algebraically closed fields induce isomorphisms of 2-completed (not rational!) stable homotopy theory, (see the Appendix), and in the case of $k=\mathbb{C}$, we have a topological realization map [19], which induces a map of Adams-Novikov spectral sequences

$$
\phi: E_{M o t, r}^{s, m+n \alpha} \rightarrow E_{r}^{s, m+n}
$$

where the left (resp. right) hand side of (37) denotes the Motivic (resp. topological) Adams-Novikov spectral sequence $r$-terms. It is useful to note the following

Lemma 15. $E_{M o t, r}$ is of the form

$$
G[\theta] / H[\theta]
$$

where $G$ is spanned (as an abelian group) by elements

$$
x_{i} \in E_{M o t, r}^{s_{i}, n_{i}(1+\alpha)}
$$

and $H$ is spanned (as an abelian group) by elements

$$
y_{j} \in E_{M o t, r}^{q_{j}, m_{j}(1+\alpha)+k_{j}(1-\alpha)}, 0 \leq k_{j} \leq\left\lfloor\frac{r-2}{2}\right\rfloor .
$$

Further, for any element $x \in E_{M o t, r-1}$,

$$
d_{r-1}(\phi(x))=0 \Rightarrow d_{r-1}(x)=0 .
$$

Proof: Induction on $r$. For $r=2$, the statement is observed above (we have $H=0)$. Suppose the statement is true for a given $r$. Then by (38), the following map is an isomorphism:

$$
\theta^{k}: E_{M o t, r}^{s_{i}+r, n_{i}(1+\alpha)+r-1} \rightarrow E_{M o t, r}^{s_{i}+r, n_{i}(1+\alpha)+r-1+k(1-\alpha)} .
$$

(This is because $\frac{r-1}{2} \geq\left\lfloor\frac{r-2}{2}\right\rfloor$.) Thus,

$$
\phi: E_{r, M o t}^{s_{i}+r, n_{i}(1+\alpha)+r-1} \rightarrow E_{r}^{s_{i}+r+2 n_{i}+r-1}
$$

is iso, and hence

$$
d_{r} \phi\left(x_{i}\right)=0 \Rightarrow d_{r} x_{i}=0
$$

(which, among other things, proves (39) with $r$ replaced by $r+1$ ). This means that

$$
d_{r} \theta^{k} x_{i}=0 \Rightarrow d_{r} x_{i}=0,
$$

and the statement about generators of $E_{r+1}$ follows. Regarding relations, the new relations are $d_{r}\left(x_{i}\right)$, which are in

$$
E_{M o t, r+1}^{s_{i}+r, n_{i}(1+\alpha)+r-1}
$$

We have

$$
\begin{aligned}
n_{i}(1+\alpha)+r-1= & \left(n_{i}+\frac{r-1}{2}\right)(1+\alpha)+\frac{r-1}{2}(1-\alpha), \\
& \frac{r-1}{2}=\left\lfloor\frac{r-1}{2}\right\rfloor .
\end{aligned}
$$

(If the differential is nonzero, then by (40) and sparsity, $r-1$ has to be even.) This completes the induction step.

Proof of Theorem 1: The proof of the fact that $\theta$ is a permanent cycle is relegated to Lemma 23 in the Appendix. 
Now in topology, the first non-trivial differential in the ANSS at $p=2$ outside the $\alpha$-family is

$$
d_{3}: \operatorname{Ext}_{B P_{*}}^{2,28} \rightarrow \operatorname{Ext}_{B P_{*}}^{5,30} .
$$

By the comparison, then, in the motivic case over an algebraically closed field, we must have a non-trivial

$$
d_{3}: \operatorname{Ext}_{B P G L_{*}}^{2,14(1+\alpha)} \rightarrow \operatorname{Ext}_{B P G L_{*}}^{5,14(1+\alpha)+2} .
$$

We see that the target of (42) is $\theta$ times the generator of $\operatorname{Ext}_{B P G L_{*}}^{5,15(1+\alpha)}$ corresponding to the target $\alpha_{1}^{2} \eta_{3 / 2}$ of (41). The examples in the $\alpha$ family are treated analogously, using the differentials originating in $\alpha_{4 k+2}, \alpha_{4 k+3}$. Note that by Lemma 15, any nonzero differential in the motivic ANSS must have non-zero images in the topological ANSS. Thus, in particular, we can conclude that $\alpha_{4 k}, \alpha_{4 k+1}$ are permanent cycles, and $\theta$-torsion elements are not hit by differentials. This concludes the proof of Theorem 1.

\section{The 2-COMPLETE $J$-HOMOMORPHISM}

Proof of Lemma 2: First we note that on the level of homotopy categories, $P F_{s}$ and $F P_{s}$ both preserve 2-equivalences. To this end, a 2-equivalence is the same thing as an equivalence after smashing with $M \mathbb{Z} / 2$, which is the same as an equivalence on the level of cofibers of the map 2. But since $P F_{s}$ and $F P_{s}$ preserve cofibrations and 2, they preserve 2-equivalences.

Thus, we know that for a motivic spectrum $X$, the canonical map

$$
F P_{s}(X) \rightarrow F P_{s}\left(X_{2}^{\wedge}\right)
$$

is a 2-equivalence. On the other hand, if $Y \rightarrow Z$ is a 2-equivalence, then

$$
P F_{s} Y \rightarrow P F_{s} Z
$$

is a 2-equivalence, so the induced map on homotopy classes

$$
\left[P F_{s} Z, X_{2}^{\wedge}\right] \rightarrow\left[P F_{s} Y, X_{2}^{\wedge}\right]
$$

is an isomorphism. But by adjunction, (43) is the same thing as

$$
\left[Z, F P_{s}\left(X_{2}^{\wedge}\right)\right] \rightarrow\left[Y, F P_{s}\left(X_{2}^{\wedge}\right)\right],
$$

so $F P_{s}\left(X_{2}^{\wedge}\right)$ is 2-complete.

Now similarly to the $J$-homomorphism calculation in the topological case, the proof of Theorem 3 has two different parts, detection and vanishing. For both parts we must use a certain ingenuity to mimic the corresponding arguments in topology. To tackle the detection part first, it is tempting to use the version of the ANSS described above, mimicking the Thom spectra approach outlined in Ravenel [22]. The difficulty is however that the element in $\operatorname{Im} J_{\mathbb{C}}$ in dimension $1 \bmod 8$ is detected in $E x t^{3}$, so the Thom space construction does not work immediately, and a more elaborate argument is needed there.

For an inclusion of fields $i^{O p}: K \subset L$, (the reason for the notation is that we are formally thinking of $i: \operatorname{Spec}(L) \rightarrow \operatorname{Spec}(K)$, although this is not in the motivic category), we have the base change functor $i^{*}$ from $\mathbb{A}^{1}$-spaces over $K$ to $\mathbb{A}^{1}$-spaces over $L$ which commutes with pushforward. (Similarly for spectra.) Clearly, $i^{*}$ has 
a right adjoint, which we will denote by $i_{*}$, and preserves cofibrations and cofibration equivalences, so it is a Quillen adjunction, and on the homotopy category, $i_{*}$ commutes with FP. A similar argument also applies to the $M \mathbb{Z} / 2$-localized $\mathbb{P}^{1}$-stable categories. Now on $M \mathbb{Z} / 2$-localized $\mathbb{P}^{1}$-stable categories, when both $K$, $L$ are algebraically closed, $i^{*}$ induces iso in $\bmod 2$ motivic homology, and hence by our spectral sequence on $\bmod 2$ homotopy of finite homotopy colimits of varieties. Hence, by a colimit argument, $i^{*}$ induces an iso on homotopy groups in the $M \mathbb{Z} / 2$-localized $\mathbb{P}^{1}$-stable categories, but so does $i_{*}$ (by the commutation with $F P)$. Thus, $i^{*}$ and $i_{*}$ are inverse equivalences of $M \mathbb{Z} / 2$-localized $\mathbb{P}^{1}$-stable categories. This means that we may change fields to work in an algebraically closed field of our choice, say, $\mathbb{C}$.

But then we have the ordinary topological realization functor |?|, under which the map (3) becomes just the ordinary complex $J$-homomorphism (at least when we compose with the canonical map $\left.\left|F_{M o t}\right| \rightarrow F\right)$. Furthermore, clearly we have a natural equivalence

$$
X \stackrel{\simeq}{\longrightarrow}|P F(X)|
$$

Now consider the diagram

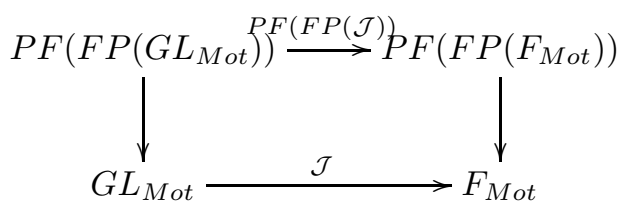

where the vertical maps are adjunction counits. Applying topological realization to $(46)$, we then get

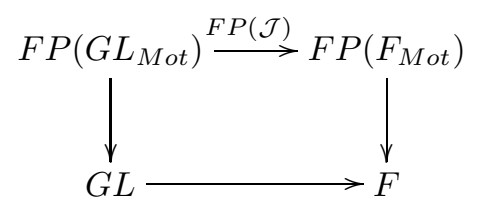

where the bottom row is the ordinary topological $J$-map. Thus, if we know that the left vertical arrow of (47) is an iso in homotopy after 2-completion, we are done by comparison with the ordinary topological $J$-homomorphism.

The key point is that we analogously have a natural map

$$
F P(E) \rightarrow|E|
$$

for a $\mathbb{P}^{1}$-spectrum $E$, and since taking the 0 -space of a spectrum obviously commutes with topological realization, the left vertical arrow of (47) coincides with the map obtained by passing to -1 -spaces of the natural map

$$
F P\left(K_{M o t}\right) \rightarrow\left|K_{M o t}\right| .
$$

But this map is just the usual map from algebraic to topological $K$-theory spectra. In effect, on 0 -components of the 0 -spaces, if we let

$$
B G L_{d i s c} \rightarrow F P B G L_{M o t}
$$

be the +-construction $\left(G L_{\text {disc }}\right.$ is just the direct limit of the general linear groups of the ground field, considered as a discrete group), it is the adjoint of the obvious 
map

$$
P F B G L_{d i s c} \rightarrow B G L_{M o t} .
$$

This follows by reinterpreting the proof of Proposition 3.9 of [19]. Now applying |PF?| to (49), we get a commutative diagram

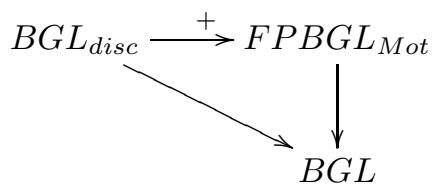

so the vertical map is the right map by universality of the +-construction.

By Suslin [23], (48) is an equivalence after 2-completion.

To prove that the elements not detected in the topological realization actually vanish in the motivic $J$-homomorphism, we mimic the method of Adams [3] in the $\mathbb{A}^{1}$-homotopy category. First, let $W=\left(S^{1+\alpha}\right)^{\times n}$. Let also $V=S^{n(1+\alpha)}$. Then there is a natural projection

$$
p: W \rightarrow V \text {. }
$$

Lemma 16. The suspension of the natural map from $V$ to the cofiber of $p$ is nullhomotopic.

Proof: First, it is a well known fact that when $Y$ has a point (a trivial assumption in spaces, but not in $\mathbb{A}^{1}$-spaces), we have

$$
X * Y \simeq X \wedge \tilde{\Sigma} Y
$$

where $\tilde{\Sigma}$ denotes the unreduced suspension and $*$ denotes the join. The obvious inclusion $X * Y \rightarrow \Sigma(X \times Y)$ along with (51) then gives a splitting (=right inverse up to homotopy) of the collapse map

$$
\Sigma(X \times Y) \rightarrow(\Sigma X \wedge Y)
$$

Therefore, for $n$ based spaces $X_{1}, \ldots, X_{n}$, each of the collapse maps

$$
\Sigma\left(X_{1} \times \ldots \times X_{n}\right) \rightarrow X_{1} \wedge \Sigma\left(X_{2} \times \ldots \times X_{n}\right) \rightarrow \ldots \rightarrow X_{1} \wedge \ldots \wedge \Sigma X_{n}
$$

has a right inverse up to homotopy, and hence so does their composition.

Now consider the diagram

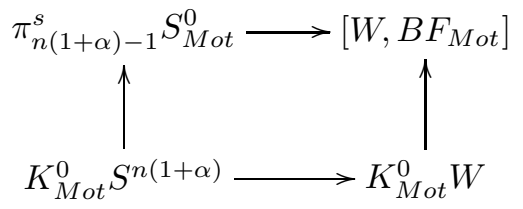

Recall from [19] that in the motivic homotopy category, $B$ is inverse to $\Omega$ in grouplike monoids. The proof there extends in a standard fashion to delooping $E_{\infty}$-spaces an arbitrary finite number of times. (What we miss in the $\mathbb{A}^{1}$-category is a device which would guarantee delooping with respect to copies of $S^{\alpha}$.) $F_{M o t}$, by mimicking the construction from topology, is still a group-like $E_{\infty}$ space, and hence can be delooped an arbitrary finite number of times. This allows us to rewrite the motivic $J$-homomorphism on $K_{M o t}^{0}$ as

$$
K_{M o t}^{0} S^{n(1+\alpha)} \rightarrow \pi_{n(1+\alpha)}^{s} B F_{M o t},
$$


which in turn can be rewritten as the left hand vertical arrow of (53). The right hand vertical arrow is the analogous map with $S^{n(1+\alpha)}$ replaced by $W$.

Lemma 17. The horizontal arrows of (53) are injective.

Proof: The arrows in question are induced by the map of $\mathbb{A}^{1}$-spaces (50). Since however the space we map into in each case can be delooped, the maps in question can be rewritten as induced by the suspension of (50). By Lemma 16, however, this suspension is a homotopy retraction.

Lemma 18. For the generator $z \in \tilde{K}_{M o t}^{0} S^{n(1+\alpha)} \cong \mathbb{Z},\left(\psi^{3}-1\right) z$ maps to 0 in the 2 -completed motivic J-homomorphism (where $\psi$ denotes the Adams operations).

Proof: We wish to completely mimic the proof of Adams [3]. By Lemma 17, we may pass to $W$, where $z$ is a sum of copies of the tautological line bundle $\xi$ over the individual copies of $S^{1+\alpha}=\mathbb{P}^{1}$. We obviously have a fiberwise degree 3 map from the associated spherical bundle (with fiber $S^{1+\alpha}$ ) of $\xi$ to the associated sphere bundle of $\xi^{3}$, simply by taking fiberwise the completion of the map $\mathbb{P}^{1} \rightarrow \mathbb{P}^{1}$ extending the 3'rd power map $\mathbb{G}_{m} \rightarrow \mathbb{G}_{m}$. On the other hand, there is also a fiberwise degree 3 map from the associated sphere bundle of $\xi+1$ (with fiber $S^{2(1+\alpha)}$ ) to itself: simply take the identity, and add it to itself three times fiberwise using the suspension coordinate. Thus, we would like to say that there is a fiberwise degree 3 map from the associated sphere bundle of the virtual bundle $\xi-\xi^{3}$ to the trivial sphere bundle. We can make precise sense of this by passing to an affinization $\mathbb{P}^{1 \prime}$ of the base space $\mathbb{P}^{1}$; then there exists a number $N$ such that $\eta=N+\xi^{\prime}-\xi^{\prime 3}$ (where $\xi^{\prime}$ is the pullback of $\xi$ ) is realized by an actual vector bundle, and we then have a fiberwise degree 3 map from the associated sphere bundle (with fiber $S^{N(1+\alpha)}$ ) to the trivial sphere bundle $\mathbb{P}^{1 \prime} \times S^{N(1+\alpha)}$. (Note that the Grothendieck-Witt ring of an algebraically closed field is $\mathbb{Z}$, so we may operate with the degree in the same way as in topology.)

Now the next step is to note that since we have a "cell decomposition" of $\mathbb{P}^{1 \prime}$, we can mimic precisely the topological proof of Adams $[2,3]$ to conclude that there is a natural number $M$ such that the associated sphere bundle of $3^{M} \eta$ (with fiber $S^{3^{M} N(1+\alpha)}$ ) has a fiberwise degree 1 map to the trivial sphere bundle $\mathbb{P}^{1 \prime} \times$ $S^{3^{M} N(1+\alpha)}$.

We are then done if we can mimic enough of the theory of classification of spherical bundles in the $\mathbb{A}^{1}$-category to conclude that

When $\tau$ is a vector bundle on $\mathbb{P}^{1 \prime}$ of dimension $m$ such that there exists a fiberwise degree 1 map from the associated sphere bundle of $\tau$ to the trivial sphere bundle, then the image of $\tau-m$ under the motivic $J$-homomorphism vanishes.

This can be done as follows: by gluing over coordinate patches, one can replace a spherical bundle by its "associated principal bundle" $\pi$ with fiber $F_{M o t}$ (each fiber $S$ is replaced by the $\mathbb{A}^{1}$-space of stable based equivalences $S \rightarrow S^{m(1+\alpha)}$; of course, we must first pass to fibrant replacements). Then having a fiberwise degree 1 map implies that the principal bundle $\pi$ has a section. But now spherical bundles coming from vector bundles are classified (i.e. pulled back from the universal bundle) by 
maps into $B F_{M o t}$ (since vector bundles of a fixed dimension are classified by maps into $\left.B G L_{M o t}\right)$. On the level of principal bundles, this means that we have a map of principal bundles

$$
\pi \rightarrow u
$$

where

$$
u: B\left(F_{M o t}, F_{M o t}, *\right) \rightarrow B F_{M o t}
$$

is the universal bundle (the projection). Since $B\left(F_{M o t}, F_{M o t}, *\right)$ is contractible, a principal bundle with a section is then necessarily classified by a map homotopic to the constant map, thus proving (54), and the Lemma.

The Adams operations on $\tilde{K}_{M o t}^{0} S^{n(1+\alpha)} \cong \mathbb{Z}$ are computed in the same way as in ordinary reduced topological $K$-theory of $S^{2 n}$, and it is well known that the vanishing of the complex $J$-homomorphism of $\psi^{3}-1$ in topology is a tight bound for the order of the image of the $J$-group in all dimensions except $6 \bmod 8$ (counting the dimensions as in diagram (53)). To translate the argument to the $\mathbb{A}^{1}$ category, it suffices to note that over an algebraically closed field, multiplying by the Tate twist induces an isomorphism on homotopy groups of the 2-completion of the $K_{M o t}$-theory spectrum, and thus the bound can be applied also after multiplying by powers of the Tate twist.

Actually, commutation with the Tate twist has to be justified. Unstably, $S^{\alpha}=$ $\mathbb{G}_{m}$ represents $H^{\alpha}(?, \mathbb{Z})$. Thus, we cannot quite conclude that the Tate twist is represented by a map $S^{1} \rightarrow S^{\alpha}$ (in fact, that is usually false), but we can conclude that this is true after 2-completion. However, (over an algebraically closed field) we do have

$$
F P\left(\mathbb{G}_{m}\right)_{2}^{\wedge} \simeq B \mathbb{Z}_{2}=\left(S^{1}\right)_{2}^{\wedge}
$$

(by comparison of homotopy groups), so for any spectrum $E$, we can conclude that the effect of $\ell$-fold Tate twist on the $n+m \alpha$ 'th homotopy groups of the 2 -completion of $E$, where $m, n-\ell \geq 0$, can be computed by the map

$$
\begin{aligned}
& \operatorname{Map}\left(S^{\ell \alpha}, \Omega^{n+(m-\ell) \alpha} E_{0}\right) \rightarrow \\
& \operatorname{Map}\left(\bigwedge_{\ell} F P\left(S^{\alpha}\right)_{2}^{\wedge}, F P\left(\Omega^{n+(m-\ell) \alpha} E_{0}\right)_{2}^{\wedge}\right) \simeq \Omega^{\ell} F P\left(\Omega^{n+(m-\ell) \alpha} E_{0}\right)_{2}^{\wedge}
\end{aligned}
$$

This is entirely an unstable construction, so it commutes with the motivic $J$-map.

The dimension in which Lemma 18 does not suffice for a tight bound of the image of $J_{M o t}$ is $n=3 \bmod 4$ (as counted in diagram (53)). In topology, this corresponds to the case of dimension $6 \bmod 8$. In this case, Adams [2] simply argues that the image of the $J$ homomorphism vanishes because it factors through the image of the real $J$-homomorphism, and the corresponding homotopy group of the orthogonal group vanishes.

We can also mimic this argument in $\mathbb{A}^{1}$-homotopy theory. We may embed

$$
G L_{n} \rightarrow O_{2 n}
$$

Over an algebraically closed field, we can consider $O_{2 n}$ as the automorphism group of the hyperbolic quadratic form

$$
x_{1} y_{1}+\ldots+x_{n} y_{n}
$$


and then $G L_{n}$ embeds by

$$
A \mapsto\left(\begin{array}{cc}
A & 0 \\
0 & \left(A^{T}\right)^{-1}
\end{array}\right)
$$

Furthermore, $O_{2 n}$ thus described acts on the affine quadric

$$
x_{1} y_{1}+\ldots+x_{n} y_{n}=1,
$$

which is homotopy equivalent to $\mathbb{A}^{n(1+\alpha)}-\{0\} \simeq S^{n(1+\alpha)-1}$ via the projection to the $x$-coordinates. This is sufficient to show that (3) factors through $O_{M o t}$, the direct limit of motivic orthogonal groups. The fact that the 2-complete homotopy groups of $O_{M o t}$ vanish in dimensions $5 \bmod 8$ now follows from the results of Hornbostel [9], (the definition of the motivic orthogonal spectrum on p. 678, and Definition 4.1 on p. 674), in conjunction with the calculations of Karoubi [13].

\section{Appendix: The motivic Adams spectral sequences}

The key point is that we focus on (bistable motivic) cell spectra [7], i.e. spectra equivalent to homotopy colimits of sequences of motivic spectra $K_{n}$, where $K_{n+1}$ is obtained from $K_{n}$ by taking the homotopy cofiber of a map

$$
\bigvee_{i \in I_{n}} S^{m_{i}} \rightarrow K_{n}
$$

where $m_{i}$ is of the form $k_{i}+\ell_{i} \alpha, k_{i}, \ell_{i} \in \mathbb{Z}$, and $S^{m_{i}}$ here denotes the $m_{i}{ }^{\text {'th }}$ suspension of the motivic sphere spectrum. We call a motivic cell spectrum of finite type if the $k_{i}$ 's are bounded below and there are only finitely many cells for each $k_{i}$.

Definition: We call a motivic spectrum $K$ of weakly finite type if $K$ is a homotopy colimit of a sequence of motivic spectra $K_{(n)}$ where we have cofibration sequences

$$
A_{(n)} \rightarrow K_{(n)} \rightarrow K_{(n+1)}
$$

where $A_{(n)}$ is equivalent to a wedge summand of a cell spectrum $B_{(n)}$ with finitely many cells, and if we denote by $k_{i}+\ell_{i} \alpha$ the dimensions of the cells of the cell spectrum

$$
\bigvee_{n} B_{(n)}
$$

then the $k_{i}$ 's are bounded below, and a given $k$ can be equal to $k_{i}$ for only finitely many values of $i$.

Lemma 19. Suppose a motivic spectrum $X$ of weakly finite type satisfies $0=2^{N}$ : $X \rightarrow X$, and also $0=\eta^{N}: \Sigma^{N \alpha} X \rightarrow X$ for some $N$. Then $X$ is of finite type.

Proof: First note that for a motivic cell spectrum $X$ of finite type with no cells of dimension $m^{\prime}+n^{\prime} \alpha, m^{\prime}<m$, we may form a cofiber sequence

$$
X(m) \rightarrow X \rightarrow X^{\prime}
$$

where $X(m), X^{\prime}$ are cell spectra, $X(m)$ has cells of dimension $m+n \alpha$ (with varying $n$ ) only, and $X^{\prime}$ has no cells of dimension $m^{\prime}+n \alpha, m^{\prime} \leq m$. Further, the spectrum 
$X(m)$ is unique up to equivalence: this is a formal consequence of the fact that $\pi_{m+n \alpha} S^{0}=0$ for $m<0$ (cf. Morel [18]) - we will use this construction again in the proof of Lemma 24 below.

We note that the construction ? $(m)$ also preserves wedge sums, and hence so does the construction $(?)^{\prime}$. Thus, it suffices to prove the statement for weakly finite type cell spectra $X$ where $X=X(m)$. Next, note that the property "being a wedge summand of a cell spectrum with finitely many cells" is clearly stable under cofibers, so we may assume $X=X(m)$ is a wedge summand of a cell spectrum with finitely many cells $Y$. Finally, taking cofibers of $2^{N}$ and $\eta^{N}$ (which yields 4 copies of $X$ ), we may assume by induction on cells that $Y$ can be formed by successively attaching finitely many copies of $S^{m+n \alpha} /(\eta, 2)$ for varying values of $n$ (and the given $m$ ). Then, however, the direct sum of $\pi_{m+n \alpha}(Y)$ with varying $n$ (and the given $m$ ) is finite, and hence the same is true for $X$. Thus, there exists a spectrum $Z$ obtained by successively attaching copies of $S^{m+n \alpha}$ for varying $n$ (and the given $m$ ) finitely many times, and a map

$$
X \rightarrow Z
$$

which induces an isomorphism in $\pi_{m+* \alpha}$. Since both spectra (61) contain only cells of dimension $m+n \alpha$ with varying $n$ (and the given $m$ ), it follows formally that (61) is an equivalence.

Corollary 20. $H \mathbb{Z} / 2$ is a cell spectrum of finite type.

Proof: A direct consequence of Lemma 19 and the proof of Proposition 14.

Define very weak equivalence (also known as slice equivalence) of motivic spectra as a map which induces isomorphism in $\pi_{k+\ell \alpha}$ for all $k, \ell \in \mathbb{Z}$. For formal reasons, ([7]), a very weak equivalence of motivic cell spectra is an equivalence. Also for formal reasons, we have cell approximation, i.e. for every motivic spectrum $X$ there exists a very weak equivalence $X^{\prime} \rightarrow X$ where $X^{\prime}$ is cell. Now we can define 2-localization in the category of cell spectra. For formal reasons, it is the same as the cell approximation of 2-localization.

Now for a spectrum $X$, the Adams resolution is the semicosimplicial object (without degeneracies)

$$
X \wedge H \mathbb{Z} / 2^{M o t} \wedge \ldots \wedge H \mathbb{Z} / 2^{M o t}
$$

where cofaces are given by the unit $S \rightarrow H \mathbb{Z} / 2^{M o t}$. If we are working in a foundational setup where $H \mathbb{Z} / 2$ is a rigid ring spectrum, we can make (62) a cosimplicial object where the codegeneracies are given by the multiplication $H \mathbb{Z} / 2^{M o t} \wedge$ $H \mathbb{Z} / 2^{M o t} \rightarrow H \mathbb{Z} / 2^{M o t}$. However, that is not important to us. There is an obvious canonical map $\phi$ from $X$ to the cosimplicial realization of its Adams resolution $X^{A d}$. Recall that throughout this paper, we work over an algebraically closed field of characteristic 0 .

Lemma 21. The canonical map

$$
\Psi: M \mathbb{Z} / 2 \rightarrow \operatorname{holim}(M \mathbb{Z} / 2) / \eta^{n}
$$

induces an isomorphism in $\pi_{*}$. 
Proof: The homotopy fiber of the map $\Psi$ is the homotopy limit of the inverse system

$$
\cdots \longrightarrow \Sigma^{(k+1) \alpha} M \mathbb{Z} / 2 \stackrel{\eta}{\longrightarrow} \Sigma^{k \alpha} M \mathbb{Z} / 2 \longrightarrow \cdots .
$$

We will prove the statement by proving that both the $\lim$ and the $\lim ^{1}$ of the homotopy groups $\pi_{m+n \alpha}$ of (63) are 0 . First, note that $\overleftarrow{(63)}$ is $\alpha$-periodic, so it suffices to consider $n=0$. Now we claim that for $k>m \in \mathbb{Z}$,

$$
\pi_{m} \Sigma^{k \alpha} M \mathbb{Z} / 2=0 .
$$

Clearly, this implies what we need. To prove (64), note that $\Sigma^{k \alpha} M \mathbb{Z} / 2$ is a pushforward of an $S^{1}$-spectrum $X$ : we work here with $\left(\mathbb{A}^{1}\right.$-local) $S^{1}$-spectra as considered in [18] (i.e. where we stabilize only with respect to $S^{1}$ and not $S^{\alpha}$ ). The integral motivic homology of its pushforward are $\mathbb{Z} / 2$ in dimensions $p+q \alpha, p+q=k$, $p \geq 0$, and 0 elsewhere. (This is where we use the fact that $K$ is an algebraically closed field.) Hence, $H_{p}^{M o t} X=0$ for $p<k$, and hence $\pi_{p} X=0$ for $p<k$ by the Hurewicz theorem 4.3.2 of [18], and hence the same is true with $X$ replaced by its pushforward $\Sigma^{k \alpha} M \mathbb{Z} / 2$ by Remark 5.3.2 of [18]. This proves (64), and thereby concludes our argument.

A cell spectrum $X$ with cells only in dimensions $m+n \alpha, n \geq 0$, can be considered as an $S^{1}$-spectrum. We will call this a cell $S^{1}$-spectrum. As observed in [18], $H A^{M o t}$ for an abelian group $A$ can also be considered as an $S^{1}$-spectrum. Denote homotopy groups in the category of $S^{1}$-spectra by $\pi_{m}^{S^{1}}$. There is a stabilization functor from $S^{1}$-spectra to motivic spectra which we shall suppress from the notation. Thus, for an $S^{1}$-spectrum $X$, we may speak of homotopy groups $\pi_{m}^{S^{1}} X$ and $\pi_{m} X$, the latter of which refer to homotopy groups of the pushforward of $X$ into the category of motivic spectra.

Lemma 22. Let $X$ be a cell $S^{1}$-spectrum such that $\pi_{m}^{S^{1}}(X)=0$ for $m<k$ such that $H_{m}^{M o t}(X)$ is a finite 2-abelian group for all $m \in \mathbb{Z}$. Then the map $X \rightarrow X^{A d}$ induces an iso in $\pi_{m}$ for all $m \in \mathbb{Z}$.

Proof: By the Hurewicz theorem [18], Theorem 4.3.2, if we put $A_{k}=\pi_{k}^{S^{1}}(X)$ (a finite 2-group), then there exists a map

$$
X \rightarrow \Sigma^{k} H A_{k}
$$

which induces an isomorphism in $\pi_{k}^{S^{1}}$. Thus the cell approximation $X^{k+1}$ of the fiber in the category of $S^{1}$-spectra satisfies the hypothesis of the lemma with $k$ replaced by $k+1$. (Note that cell approximation in the $S^{1}$-category by definition induces an isomorphism of homotopy groups in integral dimensions, and hence it induces an isomorphism of homology groups also by the Hurewicz theorem [18], Theorem 4.3.2.)

By induction, then, we obtain cofiber sequences

$$
\begin{gathered}
X^{m} \rightarrow X \rightarrow X_{m}, \\
X_{m} \rightarrow X_{m-1} \rightarrow \Sigma^{m+1} H A_{m}
\end{gathered}
$$

such that $\pi_{n}^{S^{1}} X^{m}=0$ for $n<m$. Then $X \rightarrow$ holim $X_{m}$ induces iso in $\pi_{n}^{S^{1}}$, and hence, by Remark 5.3.2 of [18], also in $\pi_{n}$, for all $n \in \mathbb{Z}$. 
By commutation of homotopy inverse limits and the long exact sequence of homotopy groups (of integral dimensions), it now suffices to prove our result for $\left(H A_{k}\right)^{M o t}$ where $A_{k}$ is a finite 2-group. This is done by direct computation, which, however, by Corollary 5 , is a rehash of the corresponding computation in topology.

Example: Let us look at the 2-completion of the $\alpha$-suspension of the motivic 0sphere $\left(S^{\alpha}\right)_{2}^{\wedge}$, which is the inverse limit of the $S^{1}$-spectra $\Sigma^{\alpha} M \mathbb{Z} /\left(2^{k}\right)$. Therefore, by Lemma 22, commutation of homotopy inverse limits, and Corollary 5 , we have a convergent Adams-like spectral sequence

$$
\operatorname{Ext}_{A_{*}}(\mathbb{Z} / 2, \Sigma \mathbb{Z} / 2) \Rightarrow \pi_{*}\left(\left(S^{\alpha}\right)_{2}^{\wedge}\right)
$$

Here the $*$ denotes integral dimensions only (no twist), $A_{*}$ is the ordinary ("topological") Steenrod algebra, and $\Sigma$ is shift up by 1 . The $E_{2}$ term (66) is obtained by reading off the twist 0 part of the $\alpha$-suspension of the motivic Steenrod algebra (and its tensor products with itself).

Now (66) has the same $E_{2}$-term as the classical Adams spectral sequence (suspended by 1), which we cannot solve, but we know one thing, namely the element in the lowest dimension has no possible differential targets, and hence has to be a permanent cycle. We have therefore proved

Lemma 23. The Tate twist element $\theta \in H_{1-\alpha}$ lifts to a stable homotopy class in $\pi_{1-\alpha}\left(\left(S^{M o t}\right)_{2}^{\wedge}\right)$.

Remark: This proof of constructing an entire "twist 0 version of the motivic Adams spectral sequence" in order to construct the Tate twist in 2-complete homotopy seemed quite unnatural to us. We were therefore quite happy to find the following more geometric argument:

Consider a finite extension $L$ of $\mathbb{Q}$ containing a $2^{k}$ th, but not $2^{k+1}$ 'st, root of unity. Let $\zeta_{k}$ be the primitive $2^{k}$ 'th root of unity in $L$. Then by Morel [18], we have an element $\left[\zeta_{2^{k}}\right] \in \pi_{-\alpha} S^{0}$ in $L$-A ${ }^{1}$-stable homotopy groups. Note that by Morel [18], Lemma 6.1.2, 4,

$$
\eta([-1] \eta+2)=0
$$

and, by [18], Lemma 6.1.2, 2,

$$
[-1]([-1] \eta+2)=0 .
$$

Since, additionally, by [18], Lemma 6.1.2, 2, clearly

$$
2^{k-1}\left[\zeta_{2^{k}}\right] \equiv[-1] \bmod \eta
$$

(67) and (68) imply

$$
2^{k-1}([-1] \eta+2)\left[\zeta_{2^{k}}\right]=0 .
$$

Therefore, $([-1] \eta+2)\left[\zeta_{2^{k}}\right]$ is in the image of a class $\theta_{k} \in\left(\pi / 2^{k-1}\right)_{1-\alpha}$ under the Bockstein

$$
\beta_{2^{k-1}}:\left(\pi / 2^{k-1}\right)_{1-\alpha} \longrightarrow \pi_{-\alpha}
$$


As we increase $k$ by passing to field extensions, we obtain an inverse system of classes $\theta_{k}$

$$
\ldots \mapsto \theta_{k+1} \mapsto \theta_{k} \mapsto \ldots
$$

(observe carefully that $\theta_{k}$ is determined up to addition of a class in $\pi_{1-\alpha}$, so any difference between $\theta_{k}$ and the image of $\theta_{k+1}$ may be corrected by altering $\left.\theta_{k+1}\right)$. The inverse limit of the system (71) then gives a class $\theta \in \pi_{1-\alpha}\left(\left(S^{0}\right)_{2}^{\wedge}\right)$, defined over any extension of $\mathbb{Q}$ containing all $\zeta_{2^{k}}$. To identify this class as the Tate twist, let us compute its image in homology: in homology,

$$
[-1] \eta+2=2,
$$

so

$$
([-1] \eta+2)\left[\zeta_{2^{k}}\right]=2\left[\zeta_{2^{k}}\right] .
$$

It follows that $\theta_{k}$ is the $\bmod 2^{k-1}$ reduction of the class $\lambda \in\left(H / 2^{k}\right)_{1-\alpha}^{M o t} \cong \mathbb{Z} /\left(2^{k}\right)$ whose $\beta_{2^{k}}$-image is $\left[\zeta_{2^{k}}\right]$. Since $\left[\zeta_{2^{k}}\right]$ generates a $\mathbb{Z} / 2^{k}$-summand of $H_{-\alpha}=K_{M}^{1}(L)$, $\lambda$ must be a unit times the Tate twist. Note that this argument is similar to Quillen's construction [21] of the Bott class in $\ell$-completed algebraic $K$-theory of $\overline{\mathbb{F}_{p}}, p \neq \ell$.

Lemma 24. Suppose $X$ is a finite type motivic cell spectrum such that $2^{N}: X \rightarrow$ $X, \eta^{N}: \Sigma^{N \alpha} X \rightarrow X$ are 0 for some $N$. Then for each $k \in \mathbb{Z}$,

$$
\pi_{k+* \alpha}(X):=\bigoplus_{\ell \in \mathbb{Z}} \pi_{k+\ell \alpha}(X)
$$

is finite.

Proof: Suppose $X$ has no cells of dimension $m+n \alpha, m<k$. First, we claim that $\pi_{k+* \alpha}(X)$ is finite. In effect, by Morel [18], we have

$$
\pi_{* \alpha}\left(\left(S^{0}\right)_{2}^{\wedge}\right)=\mathbb{Z}_{2}[\eta] / 2 \eta .
$$

Now by the connectivity result [18], inductively on stages of construction of the cell spectum, there is a "cell subspectrum" $X(k)$ consisting of all cells of $X$ of dimension $k+\ell \alpha$. More precisely, we have a cofibration sequence

$$
X(k) \rightarrow X \rightarrow X^{\prime}
$$

where $X(k)$ is finite with cells of dimension $k+\ell \alpha$ and $X^{\prime}$ is of finite type with cells only of dimensions $m+n \alpha, m>k$. Further, by (72), there is a filtration

$$
0=F_{-N \alpha}(X(k)) \ldots \stackrel{f_{n \alpha}}{\longrightarrow} F_{n \alpha}(X(k)) \stackrel{f_{(n+1) \alpha}}{\longrightarrow} F_{(n+1) \alpha}(X(k))
$$

$$
\cdots \longrightarrow F_{N \alpha}(X(k))=X(k)
$$

such that the cofiber of $f_{n \alpha}$ is a finite cell spectrum with cells of dimensions $k+n \alpha$ only. Thus, we obtain a spectral sequence with $E_{1}$-term a finite sum of $k+n \alpha$ suspensions of (72) which converge to $\pi_{k+* \alpha} X$. If $2^{N}$ and $\eta^{N}$ are 0 , we see that the $E_{\infty}$-term must be finite.

Now starting with $X$, and taking successive fibers of maps into $\Sigma^{k+n \alpha} H \mathbb{Z} / 2^{M o t}$ for suitable $n$ 's, we can wipe out $\pi_{k+* \alpha}$ after finitely many steps. In other words, there exists a spectrum $H(k)$ which is constructed from a point by finitely many 
steps of successively taking cofibers into $\Sigma^{k+n \alpha} H \mathbb{Z} / 2^{M o t}$ 's (and hence is a finite type cell spectrum with cells only in dimensions $m+n \alpha, m \geq k$ ), and a map

$$
X \rightarrow H(k)
$$

which induces an isomorphism in $\pi_{k+* \alpha}$. In asserting this, a key step is to show that when taking a map $f$ of a spectrum into $\Sigma^{k+n \alpha} H \mathbb{Z} / 2^{M o t}$ which is onto in dimension $k+n \alpha$, the fiber does not pick up any additional homotopy groups in dimensions $k+* \alpha$ (i.e. the map induced in these dimensions by the canonical map from the fiber of $f$ to $X$ is injective in these dimensions). This is not trivial, since $H \mathbb{Z} / 2^{M o t}$ has a non-trivial homotopy group in dimension $1-\alpha$. The key point, however, is that by Lemma 23, the map induced in homotopy by $f$ is onto in this dimension.

Finally, similarly as above, we may form a "sub-cell spectrum" $\overline{H(k)}$ of $H(k)$ consisting of all cells of dimensions $k+n \alpha$ for some $n$. More precisely, again, we have a cofibration

$$
\overline{H(k)} \rightarrow H(k) \rightarrow H(k)^{\prime}
$$

where $\overline{H(k)}$ is a finite cell spectrum with cells of dimension $k+n \alpha$ only, and $H(k)^{\prime}$ is a finite type cell spectrum with cells only in dimensions $m+n \alpha, m>k$. Further, by connectivity [18] and standard arguments, we must have a homotopy commutative diagram

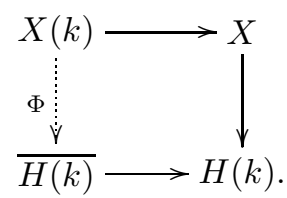

Then $\Phi$ induces an iso in $\pi_{k+* \alpha}$ and hence is an equivalence (for $X, Y$ two finite cell spectra with cells of dimension $k+n \alpha$ only, we have a spectral sequence with $E^{1}$-term consisting of $\pi_{k+n \alpha} Y$-homotopy groups, and converging to $\left.[X, Y]\right)$. Thus, we have a diagram

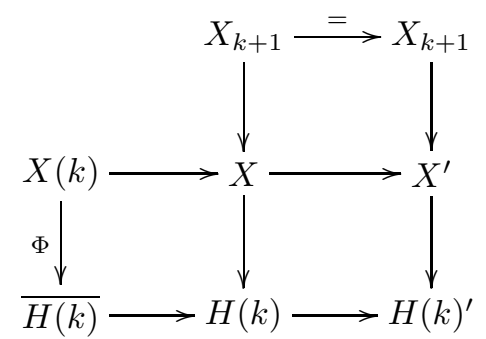

commutative up to homotopy, where the rows and columns are cofibration sequences. We see that the spectrum $X_{k+1}$ is a finite type cell spectrum with cells only of dimensions $m+n \alpha, m>k$, and whose homotopy groups differ from the homotopy groups of $X$ only by a finite group in each dimension $m+* \alpha$ (again, for given $m$, we mean the sum over all the dimensions $m+n \alpha$ ). Finally, we see that certain finite powers of $\eta$ and 2 vanish on $X_{k+1}$, since this is true for $X$ and $H \mathbb{Z} / 2^{M o t}$ (and hence $H(k)$ ). Thus, $X_{k+1}$ satisfies the same hypotheses as $X$, but with $k$ replaced by $k+1$. The resulting induction completes the proof.

The following result is standard, but we include it for completeness. 
Lemma 25. Let $X$ be a 2-complete motivic spectrum and assume that $\pi_{m+n \alpha} X \neq$ 0 . Then $\pi_{m+n \alpha}(X / 2) \neq 0$.

Proof: For any abelian group $A$, we have

$$
\operatorname{Ext}^{1}\left(\mathbb{Z} / 2^{\infty}, A\right) \cong \lim _{\longleftarrow} \operatorname{Ext}^{1}\left(\mathbb{Z} / 2^{N}, A\right) \cong \lim _{\longleftarrow} \operatorname{Ext} t^{1}\left(\mathbb{Z} / 2^{\infty}, A\right) / 2^{N} .
$$

Here the second isomorphism is by the long exact sequence in Ext associated with the short exact sequence

$$
0 \rightarrow \mathbb{Z} / 2^{N} \rightarrow \mathbb{Z} / 2^{\infty} \rightarrow \mathbb{Z} / 2^{\infty} \rightarrow 0 .
$$

The first isomorphism is by the $\lim ^{1}$ exact sequence and the fact that the $\lim ^{1}$-term vanishes, since

$$
\operatorname{Ext}^{1}\left(\mathbb{Z} / 2^{N+1}, A\right) \rightarrow \operatorname{Ext}^{1}\left(\mathbb{Z} / 2^{N}, A\right)
$$

is onto by the long exact sequence in Ext associated with the short exact sequence

$$
0 \rightarrow Z / 2^{N} \rightarrow \mathbb{Z} / 2^{N+1} \rightarrow \mathbb{Z} / 2 \rightarrow 0 .
$$

Further, by left exactness of $H o m$, we have

$$
\operatorname{Hom}\left(\mathbb{Z} / 2^{\infty}, A\right) \cong \lim \operatorname{Hom}\left(\mathbb{Z} / 2^{N}, A\right)
$$

and on the other hand

$$
\operatorname{Hom}\left(\mathbb{Z} / 2^{\infty}, A\right) / 2^{N} \subseteq \operatorname{Hom}\left(\mathbb{Z} / 2^{N}, A\right)
$$

by the long exact sequence in Ext associated with (75), so

$$
\operatorname{Hom}\left(\mathbb{Z} / 2^{\infty}, A\right) \subseteq \varliminf_{\longleftarrow} \operatorname{Hom}\left(\mathbb{Z} / 2^{\infty}, A\right) / 2^{N} .
$$

Now obviously, for any abelian group $G$,

$$
G / 2=0 \Rightarrow G / 2^{N}=0
$$

(since the premise is equivalent to $2 G=G$ ), so from (74), (76), (77) and (2), when $X$ is 2-complete,

$$
\pi_{m+n \alpha}(X) \neq 0 \Rightarrow \pi_{m+n \alpha}(X) / 2 \neq 0,
$$

but the right hand side injects into $\pi_{m+n \alpha}(X / 2)$ by the long exact sequence in homotopy associated with

$$
X \stackrel{2}{\longrightarrow} X \longrightarrow X / 2
$$

Theorem 6 now follows from the following

Lemma 26. Let $X$ be the cell approximation of the 2-completion of a cell spectrum of finite type. Then the map $\phi: X \rightarrow X^{A d}$ is a very weak equivalence.

Proof: In the present situation, we know by Morel [18] that the homotopy groups $\pi_{m+n \alpha}(X)$ vanish for $m<k$ and $\pi_{k+n \alpha}(X)$ are finitely generated $\mathbb{Z}_{2}$-modules, which further vanish for $n<\ell$ with some $\ell \in \mathbb{Z}$. Further, by Lemmas 21, 24, 25, for each $m$ there exists an $s(m)$ such that $\pi_{m+n \alpha}(X)$ vanish for $n<s(m)$.

We will construct, by induction on $m$, a sequence of spectra $X^{m}$ where $\pi_{p+q \alpha} X^{m}=$ 0 for $p<m$ and $p=m$ and $q<s(m)$ for some $s(m) \in \mathbb{Z}$, such that $\left(H \mathbb{Z} / 2^{M o t}\right)_{p+q \alpha} X^{m}$ is finite for all $p, q \in \mathbb{Z}$. Let $A_{k+\ell \alpha}=\pi_{k+\ell \alpha}(X)$. By the connectivity results of [18], 
we actually have a Hurewicz theorem and universal coefficient theorem, and thus a map

$$
X \rightarrow \Sigma^{k+\ell \alpha}\left(H A_{k+\ell \alpha}\right)^{M o t}
$$

which induces an isomorphism in $(k+\ell \alpha)^{\prime}$ th homotopy group. Let $X_{k+(\ell+1) \alpha}$ be the cell approximation of the homotopy fiber of (78). Again, by Lemma 23, this fiber does not pick up any additional homotopy groups in dimension $k+* \alpha$, similarly as in the proof of Lemma 24.

By induction then, we get an inverse system of $X_{k+n \alpha}, n>\ell$, which satisfy $\pi_{p+q \alpha} X_{k+n \alpha}=0$ for $p<k$ and $p=k, q<n$, and such that $\left(H \mathbb{Z} / 2^{M o t}\right)_{p+q \alpha} X_{k+n \alpha}$ is finite in all dimensions $p, q$. We let

$$
X^{k+1}=\underset{\leftarrow}{\operatorname{holim}} X_{k+n \alpha}
$$

We note here two points: The homotopy limit commutes, in this case, with $H \mathbb{Z} / 2^{\text {Mot }}$ homology by connectivity and the fact that $H \mathbb{Z} / 2^{M o t}$ is cell of finite type. For the same reason, cell approximation always preserves $H \mathbb{Z} / 2^{M o t}$-homology. By these arguments, and the fact that the Steenrod algebra is of finite type, $X^{k+1}$ satisfies the induction hypothesis. Because of stability, by iterating this procedure we obtain cofibration sequences

$$
\begin{gathered}
X^{m} \rightarrow X \rightarrow X_{m} \\
X_{m} \rightarrow X_{m-1} \rightarrow \Sigma \mathfrak{A}_{m}
\end{gathered}
$$

where the right hand map (79) induces isomorphism on homotopy groups in dimensions $p+n \alpha, p \leq m$, and $X_{m}$ has only non-trivial homotopy groups in dimensions $p+n \alpha, k \leq p \leq m$. In (80), $\mathfrak{A}_{m}$ is the homotopy limit of a tower of motivic Eilenberg-MacLane spectra in dimensions $m+n \alpha$, as above. Note that the induced

map from $X$ to holim $X_{m}$ induces iso in homotopy group, i.e. is a very weak equivalence.

On the other hand, by commutation of homotopy inverse limits and the long exact sequence of homotopy groups, it now suffices to prove our result for $\left(H A_{k}\right)^{M o t}$ where $A_{k}$ is a finite-dimensional $\mathbb{Z}_{2}$-module. This is done by direct computation, which, however, by Corollary 5 , is a rehash of the corresponding computation in topology, carrying along the generator $\theta$.

To prove Theorem 8, we may now consider the $B P G L$-based Adams resolution of $S^{M o t}, 2$-completing every term. Now replacing every term by its $H \mathbb{Z} / 2^{M o t}$ based Adams resolution gives a very weak equivalence by Theorem 6 , but at the same time the induced map from $\left(S_{M o t}^{0}\right)_{2}^{\wedge}$ to this composite resolution is a very weak equivalence by the same theorem. 


\section{REFERENCES}

[1] J.F. Adams: Stable homotopy and generalised homology, Chicago Lectures in Mathematics, University of Chicago Press, 1974

[2] J.F. Adams: On the groups $J(X)$. I, Topology 2 (1963) 181-195

[3] J.F. Adams: On the groups $J(X)$. II, Topology 3 (1965) 137-171

[4] J.F. Adams: On the groups $J(X)$. IV, Topology 5 (1966) 21-71, correction: Topology 7 (1968) 331

[5] A.K.Bousfield, The localization of spectra with respect to homology, Topology 18 (1979) 257-281

[6] S.Borghesi: Algebraic Morava K-theories, Invent. Math. 151 (2003) 381-413

[7] D.Dugger, D.Isaksen: Motivic cell structures, Alg. Geom. Top. 5 (2005) 615-652

[8] D.Dugger, D.C.Isaksen: The motivic Adams spectral sequence, arXiv:0901.1632

[9] J.Hornbostel: $\mathbb{A}^{1}$-representability of hermitian $K$-theory and Witt groups, Topology 44 (2005) 661-687

[10] P.Hu, I.Kriz: Some remarks on Real and algebraic cobordism, K-theory 22 (2001) 335-366

[11] P.Hu, I.Kriz: Appendix to P.Hu: On the Picard group of the stable $\mathbb{A}^{1}$-homotopy category, Topology 44 (2005) 609-640

[12] D.Isaksen: Private communication

[13] M.Karoubi: Relations between algebraic $K$-theory and Hermitian $K$-theory, Proceedings of the Luminy conference on algebraic K-theory (Luminy 1983), J. Pure Appl. Alg., vol. 34, 1984, pp. 259-263

[14] M.Levine, F.Morel: Algebraic Cobordism, Springer Monographs in Mathematics, Springer, Berlin, 2007

[15] M.Levine, F.Morel: Cobordisme algebrique I, C.R. Acad.Sci. Paris Ser. I Math. 332 (2001) 723-728

[16] M.Levine, F.Morel: Cobordisme algebrique II, C.R. Acad.Sci. Paris Ser. I Math. 332 (2001) 815-820

[17] F.Morel: Suite spectrale d'Adams et invariants cohomologiques des formes quadratiques C.R. Acad.Sci. Paris Ser. I Math. 328 (1999) 963-968

[18] F.Morel: On the motivic stable $\pi_{0}$ of the sphere spectrum, Axiomatic, Enriched and Motivic Homotopy Theory, pp. 219-260, 2004 Kluwer Academic Publishers

[19] F. Morel, V. Voevodsky: $\mathbb{A}^{1}$-homotopy theory of schemes, Inst. Hautes Études Sci. Publ. Math. 90 (1999) 45-143

[20] M.Nakaoka: Cohomology $\bmod p$ of symmetric products of spheres I,II, J. Inst. Poly. Osaka City Univ. 9 (1958) 1-18, 10 (1959) 67-89

[21] D. Quillen: On the cohomology and $K$-theory of the general linear groups over a finite field, Ann. of Math. (2) 96 (1972) 552-586

[22] D.C.Ravenel: Complex cobordism and the stable homotopy groups of spheres, Pure and Applied Mathematics, vol. 121, Academic Press Inc., Orlando, FL, 1986

[23] A. Suslin: On the $K$-theory of algebraically closed fields, Invent. Math. 73 (1983) 241-245

[24] V.Voevodsky: On the zero slice of the sphere spectrum, T. Mat. Inst. Steklova 246 (2004) 106-115, Proc. Steklov Inst. Math. 246 (2004), 93-102

[25] V.Voevodsky: Reduced power operations in motivic cohomology, Publ. Math. Inst. Hautes Etudes Sci. 998 (2003) 1-57

[26] V. Voevodsky: Motivic Eilenberg-MacLane spaces, preprint, UIUC $K$-theory archive, 846

[27] V. Voevodsky: Unstable motivic homotopy categories in Nisnevich and cdh-topologies, preprint, UIUC $K$-theory archive, 444 